\title{
FitorRemediação do Herbicida TRIFLOXYSUlFuron SODIUM ${ }^{1}$
}

\author{
Phytoremediation of the Herbicide Trifloxysulfuron Sodium
}

\author{
SANTOS, J.B. ${ }^{2}$, PROCÓPIO, S.O. ${ }^{3}$, SILVA, A.A. ${ }^{4}$, PIRES, F.R. ${ }^{5}$, RIBEIRO JÚNIOR, J.I. ${ }^{6}$, \\ SANTOS, E.A. ${ }^{7}$ e FERREIRA, L.R. ${ }^{4}$
}

\begin{abstract}
RESUMO - Este trabalho teve como objetivo avaliar a eficiência de espécies vegetais na fitorremediação do herbicida trifloxysulfuron sodium em solos, utilizando o milho como planta indicadora. Os tratamentos foram compostos pela combinação de espécies (Calopogonium muconoides, Crotalaria juncea, Crotalaria spectabilis, Vicia sativa, Cajanus cajan, Canavalia ensiformis, Helianthus annus, Dolichus lablab, Pennisetum glaucum, Stylosantes guianensis, Mucuna deeringiana, Mucuna cinereum, Mucuna aterrima, Raphanus sativus e Lupinus albus), semeadas anteriormente à cultura do milho, mais um tratamento controle (sem cultivo prévio) e três doses do herbicida trifloxysulfuron sodium $\left(0,00 ; 3,75\right.$; e 15,00 $\left.\mathrm{g} \mathrm{ha}^{-1}\right)$. O delineamento experimental usado foi o de blocos ao acaso, em esquema fatorial, sendo utilizadas três repetições. A semeadura das espécies vegetais nos vasos foi feita no dia seguinte à aplicação do trifloxysulfuron sodium. Após 80 dias da semeadura, as espécies vegetais foram cortadas na altura do coleto, descartando-se a sua parte aérea. A seguir, foi realizada a semeadura do milho (cultivar AG-122). Aos 45 dias após a emergência das plantas de milho foram avaliadas a altura de plantas e a sua biomassa seca da parte aérea. Verificouse que as espécies $M$. aterrima e $C$. ensiformis foram eficientes na descontaminação do herbicida trifloxysulfuron sodium em solo.
\end{abstract}

Palavras-chave: fitorremediação, descontaminação, adubos verdes, solos.

\begin{abstract}
This work aimed to evaluate the efficiency of plant species in the phytoremediation of the herbicide trifloxysulfuron sodium in soils, by using corn crop as a bioindicator. The treatments were composed by the combination of the species (Calopogonium muconoides, Crotalaria juncea, Crotalaria spectabilis, Vicia sativa, Cajanus cajan, Canavalia ensiformis, Helianthus annus, Dolichus lablab, Pennisetum glaucum, Stylosantes guianensis, Mucuna deeringiana, Mucuna cinereum, Mucuna aterrima, Raphanus sativus and Lupinus albus) sowed before the corn, without previous cultivation (control), and three rates of trifloxysulfuron sodium $\left(0.00,3.75\right.$ and $\left.15.00 \mathrm{~g} \mathrm{ha}^{-1}\right)$. The experiment was arranged in a randomized block design, in a factorial scheme, with three replicates. Eighty days after sowing, the plants were cut and the shoots discarded. After that, corn (cultivar AG-122) was sowed. Forty five days after emergence, corn height and shoot dry biomass were recorded. $M$. aterrima and $C$. ensiformis were considered efficient regarding decontamination of the herbicide residues in soil.
\end{abstract}

Key words: phytoremediation, decontamination, green manure, soils.

\section{INTRODUÇÃO}

Fitorremediação é o uso de plantas e sua comunidade microbiana associada para degradar, seqüestrar ou imobilizar poluentes no solo (Siciliano \& Germida, 1999). O uso de plantas para despoluir áreas com solos contaminados apresenta elevado potencial de

1 Recebido para publicação em 6.6.2003 e na forma revisada em 18.6.2004.

2 Doutorando do Departamento de Fitotecnia da Universidade Federal de Viçosa - UFV, 36571-000 Viçosa-MG; ${ }^{3}$ Prof. do Dep. de Fitossanidade da Universidade Federal de Pelotas - UFPEL, 96010-900 Pelotas-RS. ${ }^{4}$ Prof. do Dep. de Fitotecnia da UFV; ${ }^{5}$ Prof. do Dep. de Agronomia da FESURV; ${ }^{6}$ Prof. do Dep. de Informática da UFV; ${ }^{7}$ Acadêmico de Agronomia, UFV. 
utilização em relação aos métodos convencionais, como bombeamento e tratamento, ou ao de remoção física da camada contaminada, por apresentar potencial para tratamento in situ (Perkovich et al., 1996) e ser economicamente viável (Vose et al., 2000).

Essa técnica, que no Brasil é ainda incipiente, tem seu uso difundido nos Estados Unidos e na Europa, principalmente na remediação de metais pesados, sendo identificadas algumas espécies de comprovada eficiência (Radin, 2000; Accioly \& Siqueira, 2000). Outra recente linha de pesquisa tem como objetivo selecionar plantas para a biodegradação de contaminantes orgânicos, como petróleo e seus derivados, e de pesticidas (Anderson \& Coats, 1995; Cunningham et al., 1996; Fernandez et al., 1999; Wilson et al., 1999; Vose et al., 2000).

A fitorremediação de herbicidas representa uma área de pesquisa interessante, pois esses compostos, amplamente utilizados na agricultura mundial no controle de plantas daninhas, apresentam riscos potenciais de contaminação do solo e da água (Perkovich et al., 1996). Além disso, produtos que apresentam efeito residual longo - que pode variar de alguns meses até três anos ou mais - provocam fitotoxicidade em culturas sensiveis (carryover) plantadas após a utilização desses herbicidas, como observado para atrazine e imazaquin por Siqueira et al. (1991).

O trifloxysulfuron sodium, recentemente registrado no País, é utilizado na cultura do algodão em pós-emergência inicial, porém tem apresentado problemas de carryover na cultura do feijão. Mesmo sendo um produto utilizado em baixas concentrações (em torno de $\left.7,5 \mathrm{~g} \mathrm{ha}^{-1}\right)$, o período de espera para o plantio de culturas sensiveis, recomendado pelo fabricante, é de, aproximadamente, oito meses a contar de sua aplicação. Esse fato pode limitar sua utilização em áreas onde o agricultor cultiva, por exemplo, feijão no inverno.

A identificação de espécies de plantas que consigam fitorremediar o trifloxysulfuron sodium e, ainda, reúnam caracteristicas de interesse agronômico, como as leguminosas, seria de grande interesse. O uso deste grupo de plantas, além de possibilitar a despoluição do solo, proporcionaria também, entre outros beneficios, por exemplo, a fixação simbiótica do nitrogênio atmosférico (Rogers et al., 1996).

Com a finalidade de identificar espécies potencialmente fitorremediadoras de solos contaminados por herbicidas, alguns trabalhos têm sido realizados (Boyle \& Shann, 1998; Wilson et al., 1999, 2000). Das espécies testadas, algumas têm comprovado sua eficiência na redução dos níveis de determinados herbicidas no solo. Estudos envolvendo solo rizosférico da espécie Kochia scoparia evidenciaram que a degradação de metolachlor e, principalmente, atrazine foi maior do que quando comparada à de solos não-vegetados (Anderson et al., 1994; Anderson \& Coats, 1995; Perkovich et al., 1996; Rice et al., 1997).

Objetivou-se neste trabalho avaliar a eficiência de espécies vegetais na fitorremediação do herbicida trifloxysulfuron sodium em solos, utilizando o milho como planta indicadora.

\section{MATERIAL E MÉTODOS}

O experimento foi realizado em casa de vegetação pertencente ao Departamento de Fitotecnia da Universidade Federal de Viçosa, em Viçosa-MG, sendo conduzido em vasos, contendo como substrato um solo classificado como Argissolo Vermelho-Amarelo, de textura argilo-arenosa, cuja caracterização físicoquímica encontra-se na Tabela 1.

Os tratamentos foram compostos pela combinação entre as espécies vegetais Calopogonium muconoides (calopogônio), Crotalaria juncea (crotalária), Crotalaria spectabilis (crotalária), Vicia sativa (ervilhaca), Cajanus cajan (feijão-guandu), Canavalia ensiformis (feijão-de-porco), Helianthus annus (girassol), Dolichus lablab (lablabe), Pennisetum glaucum (milheto), Stylosantes guianensis (mineirão), Mucuna deeringiana (mucuna-anã), Mucuna cinereum (mucuna-cinza), Mucuna aterrima (mucuna-preta), Raphanus sativus (nabo forrageiro) e Lupinus albus (tremoçobranco), semeadas anteriormente à cultura do milho, mais um controle (sem cultivo prévio) e três doses do herbicida trifloxysulfuron sodium $\left(0,00 ; 3,75\right.$; e $\left.15,00 \mathrm{~g} \mathrm{ha}^{-1}\right)$. A escolha das espécies foi baseada em experimentos preliminares de tolerância ao herbicida. 
Tabela 1 - Composição físico-química da camada arável $(0-20 \mathrm{~cm})$ do solo Argissolo Vermelho-Amarelo utilizado no experimento

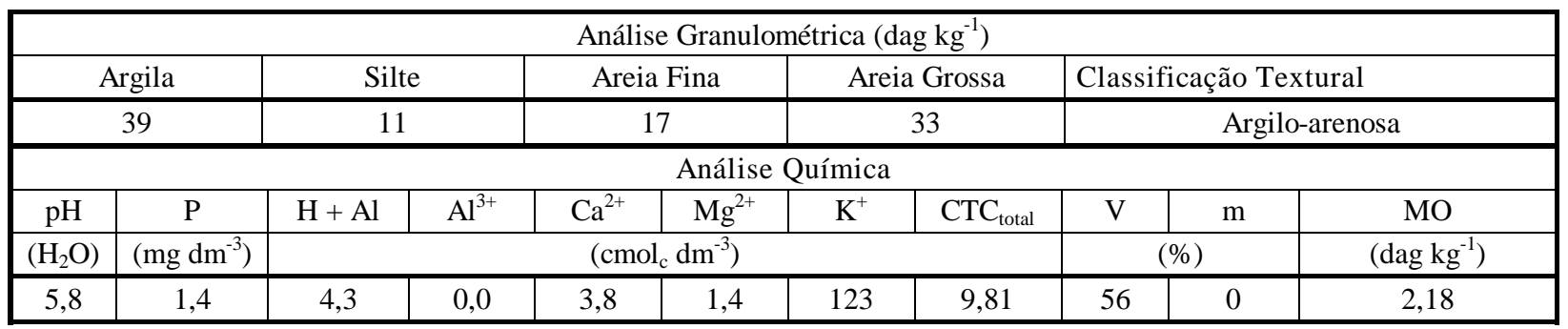

* Análises realizadas nos Laboratórios de Análises Físicas e Químicas de Solo do Departamento de Solos da UFV, segundo a metodologia da Empresa Brasileira de Pesquisa Agropecuária-EMBRAPA (1997).

O delineamento experimental utilizado foi o de blocos casualizados em esquema fatorial $16 \times 3$, com três repetições. A unidade experimental foi constituída de um vaso de polietileno, contendo $3 \mathrm{dm}^{3}$ de solo. $\mathrm{O}$ solo utilizado recebeu adubação de $600 \mathrm{~kg} \mathrm{ha}^{-1} \mathrm{da}$ fórmula 4-14-8 antes do preenchimento dos vasos. Após essa etapa, os vasos foram umedecidos e, depois de 24 horas, procedeuse à aplicação em pré-emergência do herbicida, empregando pulverizador costal pressurizado com gás carbônico $\left(\mathrm{CO}_{2}\right)$, aplicando o equivalente a $260 \mathrm{~L} \mathrm{ha}^{-1}$ de calda.

A semeadura das espécies vegetais foi realizada no dia seguinte à aplicação do trifloxysulfuron sodium. Após a emergência das plantas foi realizado desbaste, deixandose quatro vezes a densidade recomendada de cada espécie, com base no uso das espécies na adubação verde. Durante a condução do experimento os vasos foram mantidos sob irrigação.

Após 80 dias da semeadura, as espécies vegetais foram cortadas na altura do coleto, destacando-se a sua parte aérea. A seguir, foi realizada a semeadura do milho (cultivar AG-122). Após a emergência das plantas de milho, efetuou-se desbaste, deixando-se duas plantas por vaso.

Aos 45 dias após a emergência das plantas de milho foram avaliadas a altura de plantas e a biomassa seca da parte aérea das plantas, obtida por meio de pesagem do material colhido, que foi secado em estufa de circulação forçada $\left(70 \pm 2{ }^{\circ} \mathrm{C}\right)$ por 72 horas.
Todas as variáveis que atenderam às pressuposições de normalidade e homogeneidade das variâncias, por meio dos testes de Lilliefors e de Cochran, respectivamente, foram submetidas à análise de variância. O efeito entre as doses do trifloxysulfuron sodium dentro de cada espécie vegetal foi avaliado por análise de regressão, a 1 ou $5 \%$ de significância, e o efeito entre as espécies dentro de cada dose do herbicida, por meio do critério de ScottKnott, a 5\% de significância.

\section{RESULTADOS E DISCUSSÃO}

\section{Altura de Plantas}

Observou-se (Figuras 1 e 2) efeito significativo $(\mathrm{P}<0,01)$ das doses de trifloxysulfuron sodium sobre a altura das plantas de milho quando semeadas em seqüência às espécies $H$. annus, C. muconoides, C. juncea, C. spectabilis, V. sativa, C. cajan, D. lablab, P. glaucum, S. guianensis, M. deeringiana, $M$. cinereum, $L$. albus e, também, quando não houve cultivo anterior (controle), sendo suas equações de regressão apresentadas na Tabela 2. Para essas 12 espécies e para o controle, a altura das plantas diminuiu com o aumento das doses do trifloxysulfuron sodium, variando na intensidade da queda, de acordo com a espécie testada (Figuras 1 e 2), com exceção dos tratamentos contendo $H$. annus, $P$. glaucum, S. guianensis, M. deeringiana, $M$. cinereum e o controle, que acarretaram diminuição até uma determinada dose, a qual variou de acordo com o tratamento. Após essa 
dose, a altura das plantas de milho se estabilizou. Não foi detectado efeito das doses do herbicida sobre esta característica quando foram cultivadas anteriormente ao milho as espécies $C$. ensiformis, M. aterrima e $R$. sativus (Tabela 2).

Observou-se que, nos tratamentos onde não se aplicou o trifloxysulfuron sodium $\left(0,00 \mathrm{~g} \mathrm{ha}^{-1}\right)$, apresentados na Tabela 2, a única espécie semeada previamente ao milho que não afetou a altura das plantas foi $M$. aterrima. O cultivo anterior de todas as outras espécies testadas em solo livre do herbicida resultou em diminuição da altura das plantas de milho, possivelmente devido a efeitos alelopáticos.

Em relação à dose do trifloxysulfuron sodium de 3,75 $\mathrm{g} \mathrm{ha}^{-1}$, aproximadamente metade da dose comercial recomendada pelo fabricante, verificou-se que a maior altura das plantas de milho foi obtida após seu plantio em solos que receberam previamente as espécies C. ensiformis, C. spectabilis, M. aterrima, $V$. sativa e $L$. albus, sendo superior ao controle (Tabela 2).

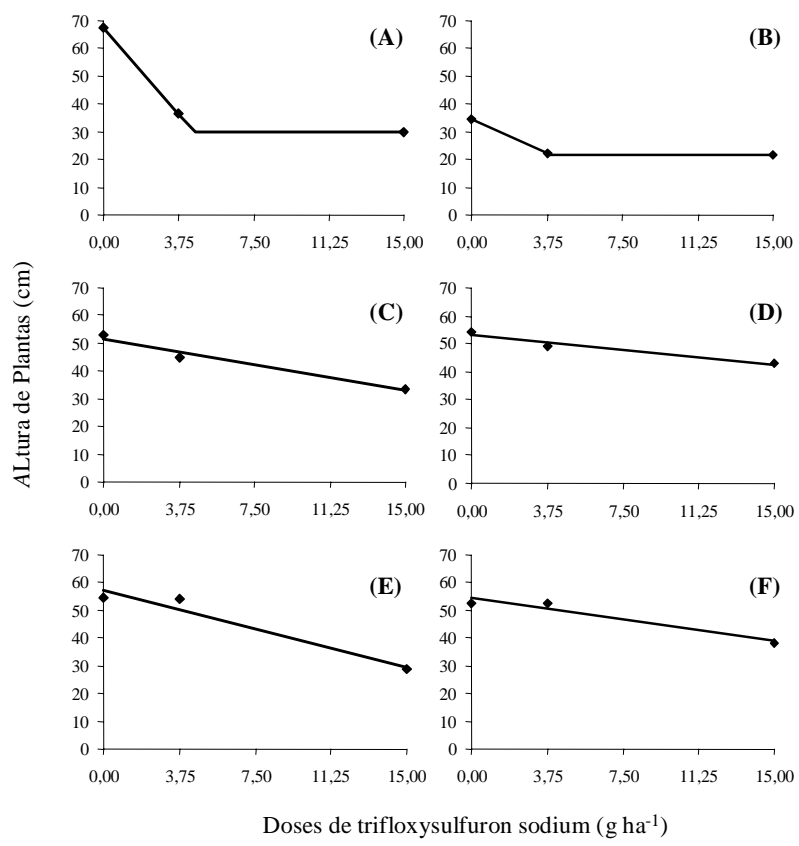

Figura 1 - Estimativa da altura de plantas de milho aos 45 dias após a emergência, após a semeadura de diversas espécies vegetais [Controle - sem cultivo (A), Helianthus annus (B), Calopogonium muconoides (C), Crotalaria juncea (D), Crotalaria spectabilis (E) e Vicia sativa (F)] em solos submetidos a três doses de trifloxysulfuron sodium.
Pela análise da Tabela 2, constata-se que, mesmo quando cultivadas em solos que receberam duas vezes a dose comercial do produto (15 $\left.\mathrm{g} \mathrm{ha}^{-1}\right)$, as plantas de milho semeadas após $M$. aterrima, C. ensiformis, $M$. cinereum e $M$. deeringiana não apresentaram queda significativa nas suas alturas, diferenciando-se das plantas cultivadas após as demais espécies, inclusive do tratamento sem cultivo prévio. Observou-se também que, nessa dose, as espécies $P$. glaucum e $H$. annus apresentaram-se significativamente inferiores ao tratamento controle, o que não foi verificado com as demais espécies. Wilson et al. (2000) constataram que a atividade de simazine em solução foi reduzida a 45 e $34 \%$, em sete dias, com a presença de Acorus gramenius e Pontederia cordata, respectivamente, sugerindo serem eficientes na fitorremediação deste herbicida.
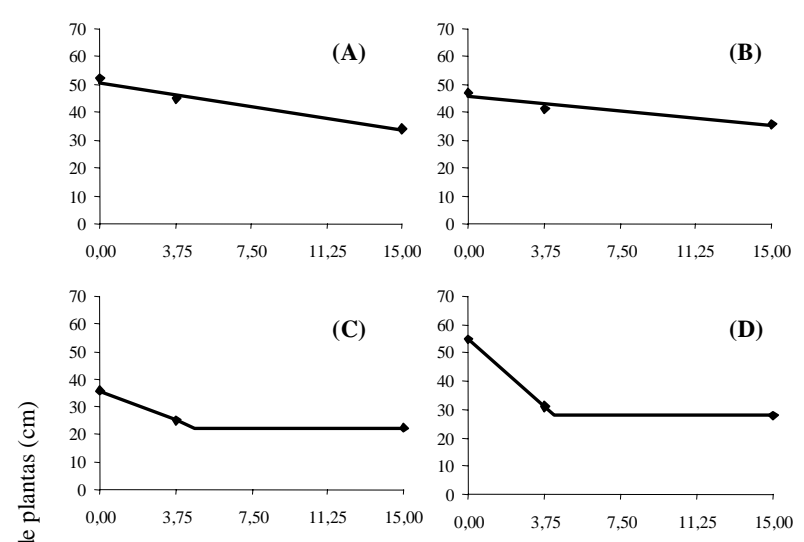

递
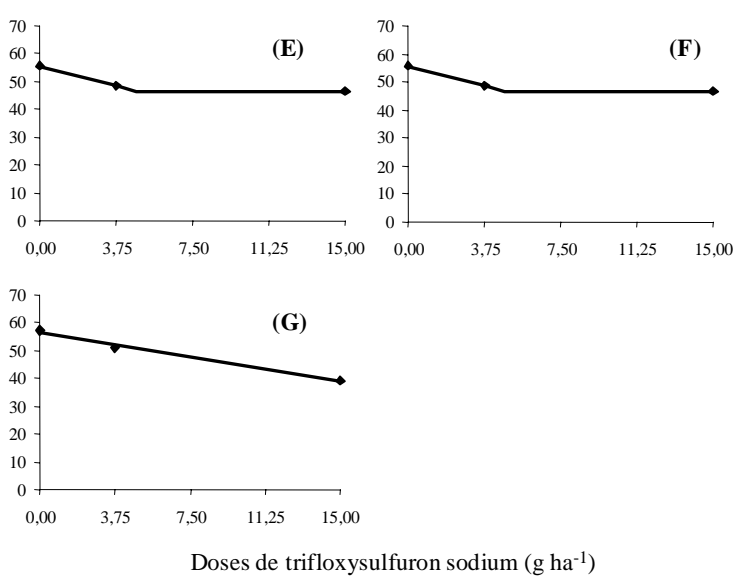

Figura 2 - Estimativas da altura de plantas de milho aos 45 dias após a emergência, após a semeadura de diversas espécies vegetais [Cajanus cajan (A), Dolichus lablab (B), Penisetum glaucum (C), Stylosantes guianensis (D), Mucuna deeringiana (E), Mucuna cinereum (F) e Lupinus albus (G)] em solos submetidos a três doses de trifloxysulfuron sodium. 
Tabela 2 - Efeito do plantio anterior de diversas espécies vegetais sobre a altura de plantas de milho aos 45 dias após a emergência, semeadas em solo submetido a três doses do herbicida trifloxysulfuron sodium (D). Viçosa-MG, 2003

\begin{tabular}{|c|c|c|c|c|c|}
\hline \multirow{3}{*}{$\begin{array}{l}\text { Espécie } \\
\text { fitorremediadora }\end{array}$} & \multicolumn{3}{|c|}{$\begin{array}{l}\text { Doses de trifloxysulfuron sodium } \\
\left(\mathrm{g} \mathrm{ha}^{-1}\right)\end{array}$} & \multirow{3}{*}{ Equação de regressão } & \multirow{3}{*}{$\mathrm{R}^{2}(\%)$} \\
\hline & 0,00 & 3,75 & 15,00 & & \\
\hline & \multicolumn{3}{|c|}{ Altura de plantas de milho $(\mathrm{cm})$} & & \\
\hline Sem cultivo & $67,33 \mathrm{a}$ & $36,67 \mathrm{c}$ & $30,00 \mathrm{c}$ & $\hat{\mathrm{Y}}=67,33-8,178^{* *} \mathrm{D}(0 \mathrm{D} \quad 4,57) \mathrm{e} \overline{\mathrm{Y}}=30,00(4,57 \mathrm{D} \quad 15)$ & 100,00 \\
\hline H. annus & $34,67 \mathrm{c}$ & $22,00 \mathrm{~d}$ & $21,67 \mathrm{~d}$ & $\hat{\mathrm{Y}}=34,66-3,378^{* * \mathrm{D}}\left(0 \mathrm{D}\right.$ 3,85) e $\overline{\mathrm{Y}}=21,67\left(\begin{array}{lll}3,85 & \mathrm{D} & 15\end{array}\right)$ & 100,00 \\
\hline C. muconoides & $53,00 \mathrm{~b}$ & $45,00 \mathrm{~b}$ & $33,33 \mathrm{c}$ & $\hat{\mathrm{Y}}=51,58-1,248^{* *} \mathrm{D}$ & 97,01 \\
\hline C. juncea & $54,33 \mathrm{~b}$ & $49,00 \mathrm{~b}$ & $42,67 \mathrm{~b}$ & $\hat{\mathrm{Y}}=53,22-0,728 * * \mathrm{D}$ & 94,73 \\
\hline C. spectabilis & $54,50 \mathrm{~b}$ & $54,00 \mathrm{a}$ & $28,67 \mathrm{c}$ & $\hat{\mathrm{Y}}=57,25-1,844^{* * \mathrm{D}}$ & 94,99 \\
\hline V. sativa & $52,67 \mathrm{~b}$ & $52,67 \mathrm{a}$ & $38,33 \mathrm{~b}$ & $\hat{\mathrm{Y}}=54,32-1,029 * * \mathrm{D}$ & 94,23 \\
\hline C. cajan & $52,00 \mathrm{~b}$ & $44,67 \mathrm{~b}$ & $34,00 \mathrm{c}$ & $\hat{\mathrm{Y}}=50,69-1,142 * * \mathrm{D}$ & 96,98 \\
\hline C. ensiformis & $54,00 \mathrm{~b}$ & $54,00 \mathrm{a}$ & $51,00 \mathrm{a}$ & $\overline{\mathrm{Y}}=53,00$ & - \\
\hline D. lablab & $47,00 \mathrm{~b}$ & $41,33 \mathrm{~b}$ & $35,33 \mathrm{c}$ & $\hat{\mathrm{Y}}=45,73-0,721 * * \mathrm{D}$ & 93,16 \\
\hline P. glaucum & $36,00 \mathrm{c}$ & $25,00 \mathrm{~d}$ & $22,33 \mathrm{~d}$ & $\hat{\mathrm{Y}}=36,00-2,933 * * \mathrm{D}(0 \mathrm{D} \quad 4,66)$ e $\overline{\mathrm{Y}}=22,33(4,66 \mathrm{D} 15)$ & 100,00 \\
\hline S. guianensis & $55,00 \mathrm{~b}$ & $31,00 \mathrm{c}$ & $28,00 \mathrm{c}$ & $\hat{\mathrm{Y}}=55,00-6,400 * * \mathrm{D}(0 \mathrm{D} \quad 4,22)$ e $\overline{\mathrm{Y}}=28,00(4,22 \mathrm{D} 15)$ & 100,00 \\
\hline M. deeringiana & $55,67 \mathrm{~b}$ & $48,33 \mathrm{~b}$ & $46,33 \mathrm{a}$ & $\hat{\mathrm{Y}}=55,66-1,956 * * \mathrm{D}(0 \mathrm{D}$ 4,77) e $\overline{\mathrm{Y}}=46,33(4,77$ D 15$)$ & 100,00 \\
\hline M. cinereum & $55,50 \mathrm{~b}$ & $48,33 \mathrm{~b}$ & $46,50 \mathrm{a}$ & 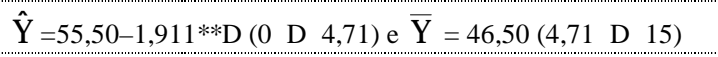 & 100,00 \\
\hline M. aterrima & $60,00 \mathrm{a}$ & $53,33 \mathrm{a}$ & $53,00 \mathrm{a}$ & $\overline{\mathrm{Y}}=55,44$ & - \\
\hline R. sativus & $35,00 \mathrm{c}$ & $30,67 \mathrm{c}$ & $30,33 \mathrm{c}$ & $\overline{\mathrm{Y}}=32,00$ & - \\
\hline L. albus & $57,17 \mathrm{~b}$ & $51,00 \mathrm{a}$ & $39,33 \mathrm{~b}$ & $\hat{\mathrm{Y}}=56,38-1,154 * * \mathrm{D}$ & 98,91 \\
\hline
\end{tabular}

Médias seguidas pela mesma letra, na coluna, não diferem entre si pelo critério de Scott-Knott $(P>0,05)$.

** Significativo pelo teste $\mathrm{t}(\mathrm{P}<0,01)$.

\section{Biomassa Seca da Parte Aérea}

Quando o milho foi semeado após a colheita das espécies C. muconoides, C. cajan, C. ensiformis, S. guianensis, M. cinereum, L. albus, D. lablab e, também, quando não houve cultivo anterior (controle), observou-se que a biomassa seca da parte aérea das plantas de milho diminuiu de forma linear $(\mathrm{P}<0,01$ ou $\mathrm{P}<0,05)$ com o aumento das doses do trifloxysulfuron sodium, variando na intensidade da queda de acordo com a espécie testada (Figura 3 e Tabela 3). Nos tratamentos contendo previamente $C$. juncea e $M$. aterrima a diminuição ocorreu até, aproximadamente, a dose estimada de 4,00 $\mathrm{g} \mathrm{ha}^{-1}$; após essa dose, a biomassa seca da parte aérea das plantas de milho tendeu a ficar constante. Não foi observado efeito das doses do herbicida sobre esta característica quando foram semeadas anteriormente à cultura do milho as espécies
$H$. annus, C. spectabilis, V. sativa, P. glaucum, $M$. deeringiana e $R$. sativus.

Verificou-se, nos tratamentos em que não se aplicou o trifloxysulfuron sodium (Tabela 3), que as únicas espécies semeadas previamente ao milho que resultaram na biomassa seca da parte aérea das plantas de milho semelhante ao tratamento controle foram C. muconoides, C. juncea e S. guianensis. Todavia, os tratamentos contendo M. aterrima e C. ensiformis se mostraram superiores ao tratamento sem cultivo prévio no que se refere a esse parâmetro. $\mathrm{O}$ cultivo anterior de todas as outras espécies testadas em solo livre do herbicida resultou em diminuição da biomassa seca da parte aérea das plantas de milho. Novamente, a razão mais provável para esses resultados seria a ocorrência de efeitos alelopáticos das plantas previamente semeadas sobre as plantas de milho, como já relatado anteriormente para a altura das plantas. 


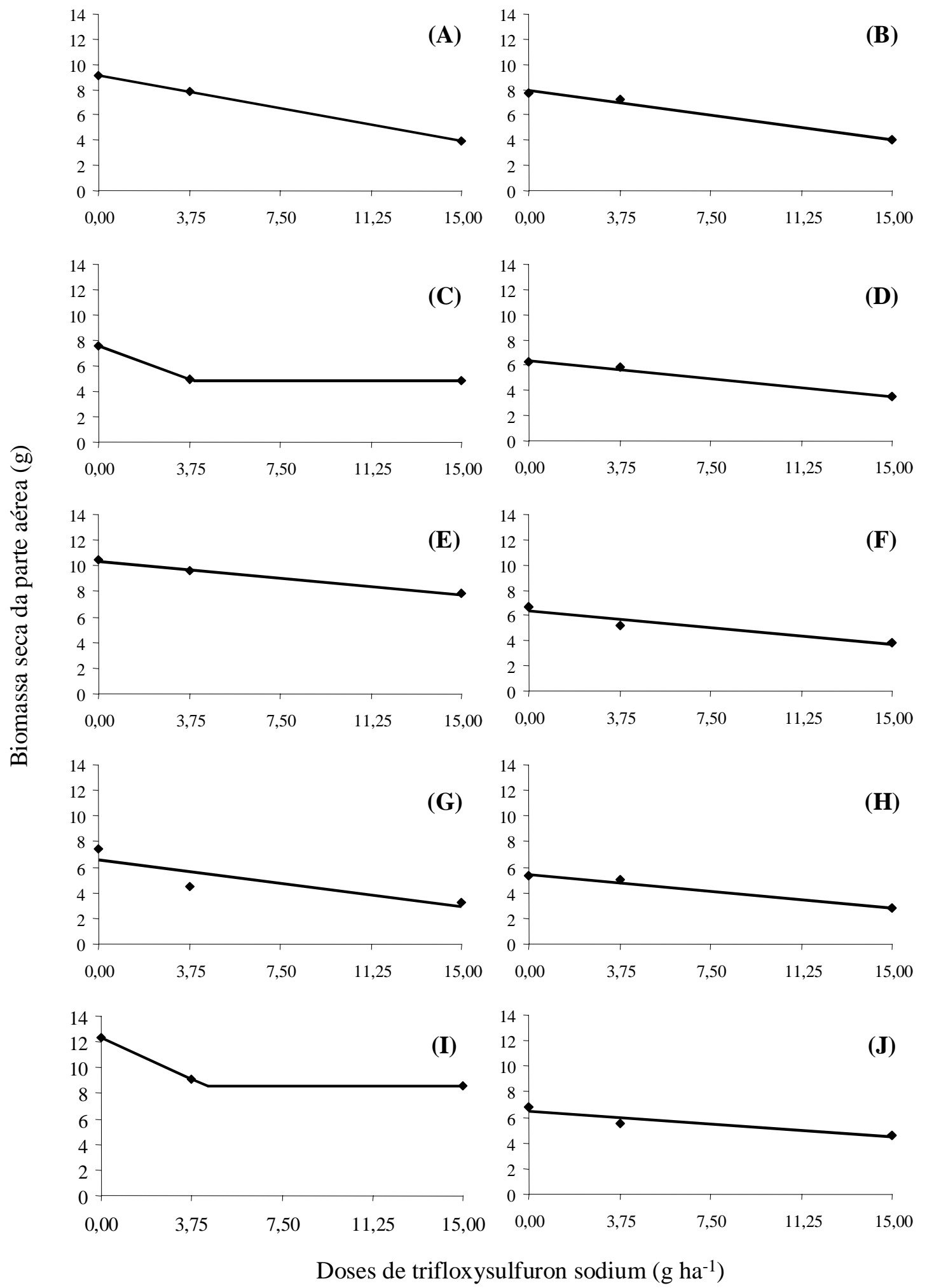

Figura 3 - Estimativas da biomassa seca da parte aérea de plantas de milho aos 45 dias após a emergência, após a semeadura de diversas espécies vegetais [Testemunha (A), Calopogonium muconoides (B), Crotalaria juncea (C), Cajanus cajan (D), Canavalia ensiformis (E), Dolichus lablab (F), Stylosantes guianensis (G), Mucuna cinereum (H), Mucuna aterrima (I) e Lupinus albus (J)] em solos submetidos a três doses de trifloxysulfuron sodium. 
Tabela 3 - Efeito do plantio anterior de diversas espécies vegetais sobre a biomassa seca da parte aérea (BSPA) de plantas de milho aos 45 dias após a emergência, semeadas em solo submetido a três doses do herbicida trifloxysulfuron sodium (D). Viçosa-MG, 2003

\begin{tabular}{|c|c|c|c|c|c|}
\hline \multirow{3}{*}{$\begin{array}{c}\text { Espécie } \\
\text { fitorremediadora }\end{array}$} & \multicolumn{3}{|c|}{$\begin{array}{l}\text { Doses de trifloxysulfuron sodium } \\
\qquad\left(\mathrm{g} \mathrm{ha}^{-1}\right)\end{array}$} & \multirow{3}{*}{ Equação de regressão } & \multirow{3}{*}{$\mathrm{R}^{2}(\%)$} \\
\hline & 0,00 & 3,75 & 15,00 & & \\
\hline & \multicolumn{3}{|c|}{ BSPA de plantas de milho $(\mathrm{g})$} & & \\
\hline Sem cultivo & $9,13 \mathrm{~b}$ & $7,80 \mathrm{a}$ & $3,98 \mathrm{~b}$ & $\hat{\mathrm{Y}}=9,11-0,343 * * \mathrm{D}$ & 99,99 \\
\hline H. annus & $3,67 \mathrm{~d}$ & $2,95 \mathrm{~b}$ & $2,26 \mathrm{~b}$ & $\bar{Y}=2,96$ & - \\
\hline C. muconoides & $7,75 \mathrm{~b}$ & $7,25 \mathrm{a}$ & $4,00 \mathrm{~b}$ & $\hat{\mathrm{Y}}=7,95-0,259 * * \mathrm{D}$ & 98,58 \\
\hline C. juncea & $7,61 \mathrm{~b}$ & $4,93 \mathrm{~b}$ & $4,81 \mathrm{~b}$ & $\hat{\mathrm{Y}}=7,61-0,714 * * \mathrm{D}(0$ D 3,92$)$ e $\overline{\mathrm{Y}}=4,81(3,92$ D 15$)$ & 100,00 \\
\hline C. spectabilis & $4,81 \mathrm{~d}$ & $3,79 \mathrm{~b}$ & $3,10 \mathrm{~b}$ & $\bar{Y}=3,90$ & - \\
\hline V. sativa & $6,31 \mathrm{c}$ & $8,26 \mathrm{a}$ & $5,10 \mathrm{~b}$ & $\overline{\mathrm{Y}}=6,56$ & - \\
\hline C. cajan & $6,29 \mathrm{c}$ & $5,79 \mathrm{~b}$ & $3,48 \mathrm{~b}$ & $\hat{\mathrm{Y}}=6,39-0,192 * * \mathrm{D}$ & 99,42 \\
\hline C. ensiformis & $10,45 \mathrm{a}$ & $9,57 \mathrm{a}$ & $7,79 \mathrm{a}$ & $\hat{\mathrm{Y}}=10,35-0,173 * \mathrm{D}$ & 99,18 \\
\hline D. lablab & $6,72 \mathrm{c}$ & $5,17 \mathrm{~b}$ & $3,79 \mathrm{~b}$ & $\hat{\mathrm{Y}}=63,43-0,179 * \mathrm{D}$ & 90,43 \\
\hline P. glaucum & $3,22 \mathrm{~d}$ & $3,87 \mathrm{~b}$ & $2,07 \mathrm{~b}$ & $\overline{\mathrm{Y}}=3,05$ & - \\
\hline S. guianensis & $7,39 \mathrm{~b}$ & $4,52 \mathrm{~b}$ & $3,24 \mathrm{~b}$ & $\hat{\mathrm{Y}}=6,54-0,239 * * \mathrm{D}$ & 77,10 \\
\hline M. deeringiana & $4,19 \mathrm{~d}$ & $3,54 \mathrm{~b}$ & $2,65 \mathrm{~b}$ & $\overline{\mathrm{Y}}=3,46$ & - \\
\hline M. cinereum & $5,33 \mathrm{c}$ & $4,97 \mathrm{~b}$ & $2,80 \mathrm{~b}$ & $\hat{\mathrm{Y}}=5,45-0,174 * \mathrm{D}$ & 98,73 \\
\hline M. aterrima & $12,34 \mathrm{a}$ & 9,07 a & $8,53 \mathrm{a}$ & $\hat{\mathrm{Y}}=12,34-0,871 * * \mathrm{D}(0 \mathrm{D} \quad 4,38)$ e $\overline{\mathrm{Y}}=8,53(4,38 \mathrm{D} \quad 15)$ & 100,00 \\
\hline R. sativus & $3,67 \mathrm{~d}$ & $3,76 \mathrm{~b}$ & $2,90 \mathrm{~b}$ & $\overline{\mathrm{Y}}=3,44$ & - \\
\hline L. albus & $6,84 \mathrm{c}$ & $5,58 \mathrm{~b}$ & $4,56 \mathrm{~b}$ & $\hat{\mathrm{Y}}=6,52-0,138 * \mathrm{D}$ & 88,72 \\
\hline
\end{tabular}

Médias seguidas pela mesma letra, na coluna, não diferem entre si pelo critério de Scott-Knott $(\mathrm{P}>0,05)$.

* Significativo pelo teste $\mathrm{t}(\mathrm{P}<0,05)$ e ** significativo pelo teste $\mathrm{t}(\mathrm{P}<0,01)$.

Quando se analisam os efeitos dos cultivos prévios na dose do trifloxysulfuron sodium de 3,75 $\mathrm{g} \mathrm{ha}^{-1}$, observa-se (Tabela 3) que a maior produção de biomassa seca da parte aérea das plantas de milho foi obtida nos solos que receberam previamente as espécies C. ensiformis, $M$. aterrima, $V$. sativa, C. muconoides, além do tratamento controle, sendo superiores a todos os outros tratamentos. Constatou-se, também, que o cultivo prévio de $M$. aterrima e C. ensiformis proporcionou as maiores biomassas de parte aérea de plantas de milho quando semeadas em solos que receberam duas vezes a dose comercial do produto (15 $\left.\mathrm{g} \mathrm{ha}^{-1}\right)$, diferenciando-se das demais espécies, inclusive do tratamento sem cultivo prévio. Anderson et al. (1994) demonstraram que a degradação de atrazine, metolachlor e trifluralin foi significativamente maior em solos rizosféricos de Kochia scoparia que em solos não-vegetados.
Conclui-se que $M$. aterrima e $C$. ensiformis foram as espécies mais eficientes na descontaminação do herbicida trifloxysulfuron sodium em solo. O uso dessa tecnologia pode resultar em maior segurança do plantio de milho em áreas onde esse herbicida tenha sido aplicado. Além disso, poderia contribuir para a redução do risco da ocorrência de impactos ambientais adversos, como a contaminação de recursos hídricos subterrâneos. No entanto, a continuação dos estudos de fitorremediação, utilizando-se essas duas espécies, é necessária, agora, em nivel de campo, visando a confirmação dos resultados obtidos.

\section{AGRADECIMENTOS}

À empresa Syngenta Proteção de Cultivos Ltda., pelo apoio financeiro.

Planta Daninha, Viçosa-MG, v.22, n.2, p.323-330, 2004 


\section{LITERATURA CITADA}

ACCIOLY, A. M. A.; SIQUEIRA, J. O. Contaminação química e biorremediação do solo. In: NOVAIS, R. F.; ALVAREZ V.; V. H.; SCHAEFER, C. E. G. R. Tópicos em ciência do solo. Viçosa: Sociedade Brasileira de Ciência do Solo, 2000. v. 1, p. 299-352.

ANDERSON, T. A.; COATS, J. R. Screening rhizosphere soil samples for the ability to mineralize elevated concentrations of atrazine and metolachlor. J. Environ. Sci. Health, v. B30, p. 473-484, 1995.

ANDERSON, T. A.; KRUGER, E. L.; COATS, J. R. Enhanced degradation of a mixture of three herbicides in the rhizosphere of a herbicide-tolerant plant. Chemosphere, v. 28 , p. $1551-1557,1994$.

BOYLE, J. J.; SHANN, J. R. The influence of planting and soil characteristis on mineralization of 2,4,5-T in rhizosphere soil. J. Environ. Qual., v. 27, p. 704-709, 1998.

CUNNINGHAM, S. D.; ANDERSON, T. A.; SCHWAB, A. P. Phytoremediation of soils contaminated with organic pollutants. Adv. Agron., v. 56, p. 55-114, 1996.

EMPRESA BRASILEIRA DE PESQUISA AGROPECUÁRIA - EMBRAPA. Centro Nacional de Pesquisa de Solos. Manual de métodos de análise de solo. 2.ed. Rio de Janeiro: 1997. 212 p.

FERNANDEZ, R. T. et al. Evaluating semiaquatic herbaceous perennials for use in herbicide phytoremediation. J. Am. Soc. Hort. Sci., v. 124, p. 539-544, 1999.

PERKOVICH, B. S. et al. Enhanced mineralization of $\left[{ }^{14} \mathrm{C}\right]$ atrazine in Kochia scoparia rhizosferic soil from a pesticidecontaminated site. Pestic. Sci., v. 46, p. 391-396, 1996.
RADIN, J. W. Using superplants to clean up our environment. Agric. Res., v.48, <http://www.ars.usda.gov/ is/AR/archive/jun00/. 2000>.

RICE, P. J.; ANDERSON, T. A.; COATS, J. R. Phytoremediation of herbicide-contaminated surface water with aquatic plants. In: Phytoremediation of soil and water contaminants, 1997, Washington, DC: ACS Symposium Series... Washington, DC: American Chemical Society, 1997. p. 133-151.

ROGERS, H. B. et al. Selection of cold-tolerant plants for growth in soils contaminated with organics. J. Soil Cont., v. 5, p. 171-186, 1996.

SICILIANO, S. D.; GERMIDA, J. J. Enhanced phytoremediation of chlorobenzoates in rhizospere soil. Soil Biol. Biochem., v. 31, p. 299-305, 1999.

SIQUEIRA, J. O.; SAFIR, G. R.; NAIR, M. G. VAmycorrhizae and mycorrhizal stimulating isoflavonoid compounds reduce plant herbicide injury. Plant Soil, v. 34, p. 233-42, 1991.

VOSE, J. M. et al. Leaf water relations and sapflow in Eastern cottonwood (Populus detoides Bartr.) trees planted for phytoremediation of a groundwater pollutant. Inter. J. Phytoremed., v. 2, p. 53-73, 2000.

WILSON, P. C.; WHITWELL, T.; KLAINE, S. J. Phytotoxicity, uptake, and distribution of ${ }^{14} \mathrm{C}$-simazine in Canna hybrida 'Yellow King Hunbert'. Environ. Toxicol. Chem., v. 18, p. 1462-1468, 1999.

WILSON, P. C.; WHITWELL, T.; KLAINE, S. J. Phytotoxicity, uptake, and distribution of ${ }^{14} \mathrm{C}$-simazine in Acorus gramenius and Pontederia cordata. Weed Sci., v. 48, p. 701-709, 2000. 